Pacific Journal of Mathematics

HoST LOCALY P PRE A ABL AN GRe 


\section{ALMOST LOCALLY PURE ABELIAN GROUPS}

\section{L. BOYer AND E. A. WALKeR}

0 . Introduction. It is the purpose of this paper to introduce and to give a preliminary investigation of almost locally pure Abelian groups [see definition 1]. For primary groups the concept of almost locally pure Abelian group coincides with that of no elements of infinite height [Theorem 9].

1. Definition. A group (= Abelian group), $G$, is almost locally pure (hereafter abbreviated a.1.p.) if for every finite set of elements $g_{1}, \cdots, g_{n}$ of $G$ there exists a finitely generated pure subgroup, $P$, of $G$ which contains $g_{1}, \cdots, g_{n}$.

2. ExAmples. Direct sums of cyclic groups are clearly a.1.p. The complete direct sum of copies of the integers is a.1.p. since by [1] every finite subset is contained in a completely decomposable direct summand and each such summand is free of finite rank.

3. Remark. If one defines a group $G$ to be locally pure if every finite subset generates a pure subgroup, then it is easy to see that $G$ is a direct sum of cyclic groups of prime order, for various primes.

\section{Theorem. A direct sum of a. 1. p. groups is a.1.p.}

Proof. Let $G=\sum_{\alpha} \oplus H_{\alpha}$, where $\oplus$ denotes the weak direct sum, and where $H_{\alpha}$ is a.1.p. for all $\alpha$. Let $g_{1}, \cdots, g_{n}$ be in $G$. Now let $H_{\beta}$ be a summand in which some $g_{i}$ has a non-zero component, and consider the components $g_{\beta_{1}}, \cdots, g_{\beta n}$ of $g_{1}, \cdots, g_{n}$ in $H_{\beta}$. In each such $H_{\beta}$ (there are only a finite number) there exists a finitely generated pure subgroup $P_{\beta}$ containing $g_{\beta_{1}}, \cdots, g_{\beta_{n}}$. Then $\sum_{\beta} \oplus P_{\beta}$ is a finitely generated pure subgroup containing $g_{1}, \cdots, g_{n}$.

5. Theorem. If $G$ is a.1.p., if $K$ is a subgroup of $G$, and if for every finite set of elements $g_{1}, \cdots, g_{n}$ of $G$, there exists a pure subgroup, $P$, of $G$ such that the group generated by $K$ and $g_{1}, \cdots, g_{n}$ is a subgroup of $P$ and $P / K$ is finitely generated, then $G / K$ is a.1.p.

If $G$ and $G / K$ are a.1.p., where $K$ is pure in $G$, then for every finite set of elements $g_{1}, \cdots, g_{n}$ of $G$, there exists a pure subgroup, $P$, of $G$ such that the group generated by $K$ and $g_{1}, \cdots, g_{n}$ is a subgroup of $P$, and $P / K$ is finitely generated.

Received October 20, 1958. In revised form December 4, 1958. 
Proof. For the proof of the first statement, assume there exists such a $P$ in $G$ for each finite set of elements of $G$. Then if $g_{1}+K, \cdots, g_{n}+K$ are elements of $G / K$, there exists a pure subgroup, $P$, of $G$ such that the group generated by $K$ and $g_{1}, \cdots, g_{n}$ is a subgroup of $P$ and $P / K$ is finitely generated. Now $P / K$ is pure in $G / K$ and $G / K$ is a.1.p.

If $G / K$ is a.1.p. and $g_{1}, \cdots, g_{n}$ are elements of $G$, then there exists a finitely generated pure subgroup, $P / K$, of $G / K$ which contains $g_{1}+K, \cdots, g_{n}+K$. The inverse image, $P$, of $P / K$ has the desired properties.

6. Corollary. If $G$ is a.1.p. and $H$ is a finitely generated subgroup, then $G / H$ is a.1.p.

7. COROLlary. If $T$ is the torsion subgroup of an a.1.p. group, then $G / T$ is a.1.p.

Proof. For $g_{1}, \cdots, g_{n}$ in $G$, let $H$ be a finitely generated pure subgroup containing $g_{1}, \cdots, g_{n}$. Then by [2], $P$, the subgroup generated by $H$ and $T$ is pure. Clearly the subgroup generated by $T$ and $g_{1}, \cdots, g_{n}$ is a subgroup of $P$ and $P / T$ is finitely generated. Hence by the theorem $G / T$ is a.1.p.

8. EXAMPLE. A strong direct sum of a.1.p. groups is not necessarily a.1.p. Consider $G=\sum_{n} \odot C\left(p^{n}\right)$, where $\bullet$ denotes the strong direct sum and $C\left(p^{n}\right)$ is cyclic of order $p^{n}$. Then if $G$ where a.1.p., $G / T$ would be a.1.p., where $T$ is the torsion subgroup. But a torsion-free a.1.p. group, $F$, is only finitely divisible (i.e. for each $x \neq 0$ in $F$ there exists a maximum positive integer, $m_{x}$, such that $m_{x} y=x$ has a solution in $F)$, whereas the element $\left(0,1 / \mathrm{p}, 0,0,1 / p^{2}, 0,0,0,1 / p^{3}, 0,0,0,0\right.$, $\left.1 / p^{4}, \cdots\right)+T$ is not zero and is divisible by all powers of $p$.

9. THEOREM. A torsion group is a.1.p. if and only if its p-components have no elements of infinite height.

Proof. This follows from the footnote on page 79 of [1] and from 4.

10. Lemma. Every subgroup of a torsion-free a.1.p. group is a.1.p.

Proof. Let $H$ be a subgroup of the torsion-free group, $G$, and let $h_{1}, \cdots, h_{n}$ be elements of $H$. Then there exists a finitely generated pure subgroup, $P$, of $G$ which contains $h_{1}, \cdots, h_{n}$. Since $P \cap H$ is a finitely generated pure subgroup of $H, H$ is a.1.p.

11. Lemma. The torsion subgroup, T, of an a.1.p. group, $G$, is a.1.p. 
Proof. The proof is similar to the proof of Lemma 10.

12. THEOREM. Every subgroup of an a.1.p. group is a.1.p.

Proof. Let $G$ be a.1.p., let $S$ be an arbitrary subgroup of $G$ and let $T$ be the torsion subgroup of $G$. By $7 G / T$ is a.1.p. and by 10 $(S \cup T) / T$ is a.1.p. Thus $S /(S \cap T)$ is a.1.p. Now let $s_{1}, \cdots, s_{n}$ be elements of $S$. Since $S /(S \cap T)$ is a.1.p. there exists a finitely generated pure subgroup, $P /(S \cap T)$, of $S /(S \cap T)$ such that $s_{1}+(S \cap T), \cdots$, $s_{n}+(S \cap T)$ are elements of $P /(S \cap T)$. Since $P /(S \cap T)$ is finitely generated and torsion-free, $P=(S \cap T) \oplus K$, where $K$ is finitely generated and torsion-free. Since $K$ is finitely generated, it is clearly a.1.p., and it follows from 11 and 9 that $S \cap T$ is a.1.p. Hence by 4, $P$ is a.1.p. and $s_{1}, \cdots, s_{n}$ are elements of $P$. Thus there exists a finitely generated pure subgroup, $P_{1}$, of $P$ containing $s_{1}, \cdots, s_{n}$. Since $S \cap T$ is a pure subgroup of $S, P$ is a pure subgroup of $S$. Hence $P_{1}$ is pure in $S$.

13. Lemma. A countable torsion-free a.1.p. group, $G$, is free.

Proof. Let $H_{1} \subset H_{2} \subset \cdots \subset H_{n} \subset \cdots$ be an ascending chain of subgroups of $G$, each having finite rank $r$. Let $h_{1}, \cdots, h_{r}$ be a maximal linearly independent subset of $H_{1}$, and hence of all the $H_{i}^{\prime} s$. Since $G$ is a.1.p., there exists a finitely generated pure subgroup $P$ containing $h_{1}, \cdots, h_{r}$. Hence each $H_{i}$ is contained in $P$. Since $P$ is free of finite rank, it satisfies the ascending chain condition, so that by Theorem $E$, page 168, of [3], $G$ is free.

14. THEOREM. If the torsion subgroup $T$, of an a.1.p. group, $G$, has countable index, then $T$ is a direct summand (and the complementary summand is free).

Proof. By $7 \mathrm{G} / \mathrm{T}$ is a.1.p., countable and torsion-free. Thus by 13 $G / T$ is free. Hence $G=T \oplus K$.

15. Lemma. A countable a.1.p. p-group, $G$, is a direct of sum cyclic groups.

Proof. By Theorem 9 this is a restatement of a theorem of Prüfer [4].

Now we prove a generalization of Prüfer's theorem.

16. Theorem, A countable a.1.p. group, $G$, is a direct sum of cyclic groups. 
Proof. Let $T$ be the torsion subgroup of $G$. Then by 14, $G=$ $T \oplus K=T_{p_{1}} \oplus \cdots \oplus K$, where $T_{p_{i}}$ is the $p_{i}$-component of $T$. Since $G$ is countable it follows from 4 and 15 that $G$ is a direct sum of cyclic groups.

17. REMARKs. From 12 and 16 it follows that every countable subgroup of an a.1.p. group is a direct sum of cyclic groups.

If one represents the group of rational numbers as a quotient group of a free group, one obtains a pure subgroup (the kernel of the mapping) of an a.1.p. group which is not a direct summand.

From 16 it follows that if $H$ is a pure subgroup of $G$ and $G / H$ is both a.1.p. and countable, then $H$ is a direct summand of $G$ and the complementary summand is a direct sum of cyclic groups.

It follows from Corollary 6 that if $T$ is the torsion subgroup of an a.1.p. group, $G$, and if $H / T$ is finitely generated then $G / H \cong(G / T) /(H / T)$ is also a.1.p.

18. THEorem. If $H$ is pure in $G$ and if $H$ and $G / H$ are a.1.p., the $G$ is a.1.p.

Proof. Let $g_{1}, \cdots, g_{n}$ be elements of $G$. Since $G / H$ is a.1.p. there exists a finitely generated pure subgroup, $L / H$, of $G / H$ which contains $g_{1}+H, \cdots, g_{n}+H$. Since $H$ is pure and $L / H$ is finitely generated, $L=H \oplus K, K$ finitely generated. Since $g_{i}$ is in $L$ for $i=1, \cdots, n$, let $g_{i}=h_{i}+k_{i}$. Since $H$ is a.1.p. let $P$ be a finitely generated pure subgroup of $H$ which contains the $h_{i}$. Now $g_{i}$ is in $P \oplus K$ and $P \oplus K$ is pure in $L$, which is pure in $G$. Hence $P \oplus K$ is a finitely generated pure subgroup of $G$ which contains the $g_{i}$. Hence $G$ is a.1.p.

19. THEOREM. Every group, $G$, has a maximal pure a.1.p. subgroup, $M$, (which may be 0 ) and 0 is the only pure a. 1. p. subgroup of $G / M$.

Proof. The existence of $M$ is easily proved by applying Zorn's lemma. If $P / M$ were a non-zero pure a.1.p. subgroup of $G / M$, then $P$ would be a pure subgroup of $G$ and by Theorem $18 P$ would by a.1.p., contradicting the maximality of $M$.

20. CoRollary. If $G$ is a p-group and $M$ is a maximal pure a.1.p. subgroup of $G$, then $G / M$ is divisible.

Proof. Otherwise $G / M=D \oplus R$, with $D$ divisible and $R$ reduced and $R$ has a finite cyclic direct summand, $P$, which is a pure a.1.p. subgroup of $G / M$, 
21. Corollary. If $G$ is a p-group and $M$ is a countable maximal pure a.1.p. subgroup, then $M$ is a basic subgroup of $G$.

Proof. By Theorem $16 M$ is a direct sum of cyclic groups and by $20 \mathrm{G} / \mathrm{M}$ is divisible. Hence $M$ is a basic subgroup of $G$.

\section{REFERENCES}

1. R. Baer, Abelian groups without elements of finite order, Duke Math. J., 3 (1937), $68-122$.

2. J. De Groot, An isomorphism criterion for completely decomposable Abelian groups, Math. Ann. 132 (1956), 328-332.

3. L. Pontrjagin, Topological Groups, Princeton University Press, 1946.

4. H. Prüfer, Untersuchungen über die Zerlegbarkeit der abzählbaren primären Abelschen, Gruppen, Math. Zeit. 17 (1923), 35-61.

New Mexico College State College 



\section{PACIFIC JOURNAL OF MATHEMATICS}

\section{EDITORS}

\section{David Gilbarg}

Stanford University

Stanford, California

\section{R. A. Beaumont}

University of Washington

Seattle 5 , Washington

\author{
A. L. Whiteman
}

University of Southern California Los Angeles 7, California

L. J. Paige

University of California

Los Angeles 24, California

\author{
E. F. BECKENBACH \\ C. E. BURGESS \\ E. HEWITT \\ A. HORN
}

\author{
V. GANAPATHY IYER \\ R. D. JAMES \\ M. S. KNEBELMAN \\ L. NACHBIN
}

ASSOCIATE EDITORS
I. NIVEN

T. G. OSTROM

H. L. ROYDEN

M. M. SCHIFFER
E. G. STRAUS

G. SZEKERES

F. WOLF

K. YOSIDA

\section{SUPPORTING INSTITUTIONS}

\author{
UNIVERSITY OF BRITISH COLUMBIA \\ CALIFORNIA INSTITUTE OF TECHNOLOGY \\ UNIVERSITY OF CALIFORNIA \\ MONTANA STATE UNIVERSITY \\ UNIVERSITY OF NEVADA \\ OREGON STATE COLLEGE \\ UNIVERSITY OF OREGON \\ OSAKA UNIVERSITY \\ UNIVERSITY OF SOUTHERN CALIFORNIA
}

\author{
STANFORD UNIVERSITY \\ UNIVERSITY OF TOKYO \\ UNIVERSITY OF UTAH \\ WASHINGTON STATE COLLEGE \\ UNIVERSITY OF WASHINGTON \\ * * * \\ AMERICAN MATHEMATICAL SOCIETY \\ CALIFORNIA RESEARCH CORPORATION \\ HUGHES AIRCRAFT COMPANY \\ SPACE TECHNOLOGY LABORATORIES
}

Mathematical papers intended for publication in the Pacific Journal of Mathematics should be typewritten (double spaced), and the author should keep a complete copy. Manuscripts may be sent to any one of the four editors. All other communications to the editors should be addressed to the managing editor, L. J. Paige at the University of California, Los Angeles 24, California.

50 reprints per author of each article are furnished free of charge; additional copies may be obtained at cost in multiples of 50 .

The Pacific Journal of Mathematics is published quarterly, in March, June, September, and December. The price per volume (4 numbers) is $\$ 12.00$; single issues, $\$ 3.50$. Back numbers are available. Special price to individual faculty members of supporting institutions and to individual members of the American Mathematical Society: $\$ 4.00$ per volume; single issues, $\$ 1.25$.

Subscriptions, orders for back numbers, and changes of address should be sent to Pacific Journal of Mathematics, 2120 Oxford Street, Berkeley 4, California.

Printed at Kokusai Bunken Insatsusha (International Academic Printing Co., Ltd.), No. 6, 2-chome, Fujimi-cho, Chiyoda-ku, Tokyo, Japan.

PUBLISHED BY PACIFIC JOURNAL OF MATHEMATICS, A NON-PROFIT CORPORATION

The Supporting Institutions listed above contribute to the cost of publication of this Journal, but they are not owners or publishers and have no responsibility for its content or policies. 


\section{Pacific Journal of Mathematics}

\section{Vol. 9, No. $2 \quad$ June, 1959}

Lee William Anderson, On the breadth and co-dimension of a topological lattice

Frank W. Anderson and Robert L. Blair, Characterizations of certain lattices

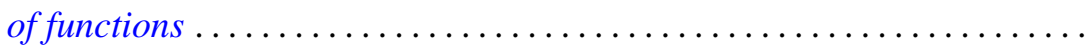

Donald Charles Benson, Extensions of a theorem of Loewner on integral

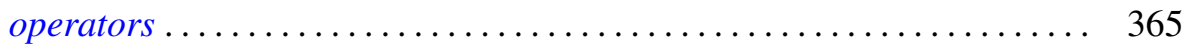

Errett Albert Bishop, A duality theorem for an arbitrary operator ........ 379

Robert McCallum Blumenthal and Ronald Kay Getoor, The asymptotic distribution of the eigenvalues for a class of Markov operators ........

Delmar L. Boyer and Elbert A. Walker, Almost locally pure Abelian

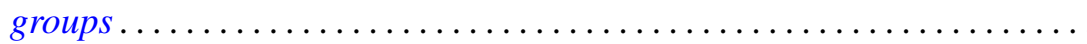

Paul Civin and Bertram Yood, Involutions on Banach algebras ........... Lincoln Kearney Durst, Exceptional real Lehmer sequences .... 415

Eldon Dyer and Allen Lowell Shields, Connectivity of topological

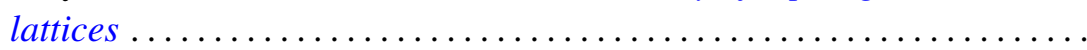

Ronald Kay Getoor, Markov operators and their associated

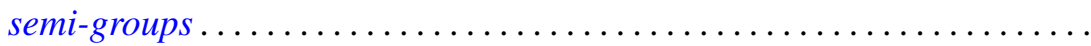

Bernard Greenspan, A bound for the orders of the components of a system of algebraic difference equations

Branko Grünbaum, On some covering and intersection properties in

Minkowski spaces ............................

Bruno Harris, Derivations of Jordan algebras ..............

Henry Berge Helson, Conjugate series in several variables.

Isidore Isaac Hirschman, Jr., A maximal problem in harmonic analysis.

II .

Alfred Horn and Robert Steinberg, Eigenvalues of the unitary part of a matrix

Edith Hirsch Luchins, On strictly semi-simple Banach algebras ...

William D. Munro, Some iterative methods for determining zeros of

functions of a complex variable...

John Rainwater, Spaces whose finest uniformity is metric .

William T. Reid, Variational aspects of generalized convex functions ....

A. Sade, Isomorphisme d'hypergroupoï des isotopes ...... . .

Isadore Manual Singer, The geometric interpretation of a special

connection . . .

Charles Andrew Swanson, Asymptotic perturbation series for characteristic

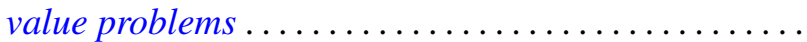

